Provided for non-commercial research and education use. Not for reproduction, distribution or commercial use.

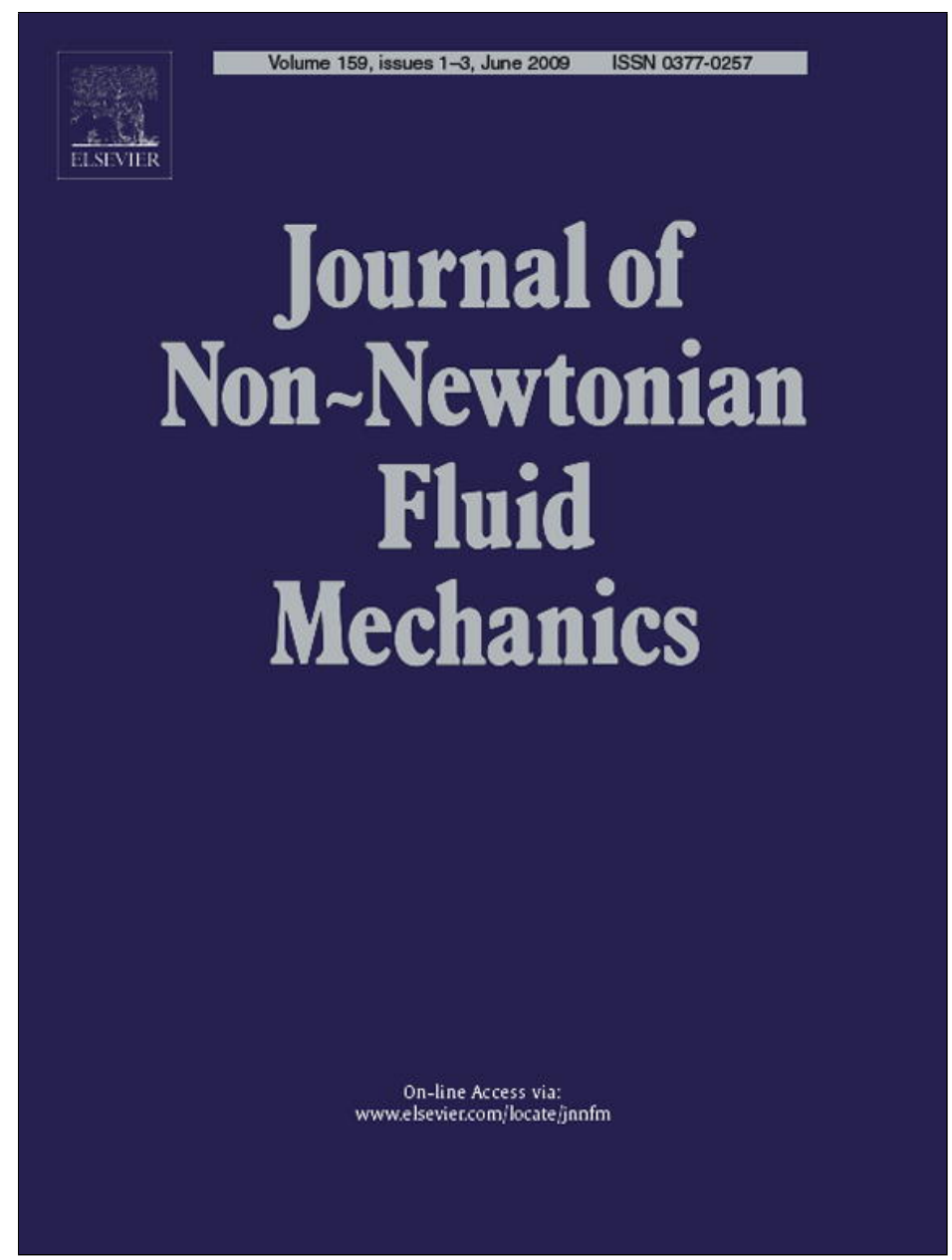

This article appeared in a journal published by Elsevier. The attached copy is furnished to the author for internal non-commercial research and education use, including for instruction at the authors institution and sharing with colleagues.

Other uses, including reproduction and distribution, or selling or licensing copies, or posting to personal, institutional or third party websites are prohibited.

In most cases authors are permitted to post their version of the article (e.g. in Word or Tex form) to their personal website or institutional repository. Authors requiring further information regarding Elsevier's archiving and manuscript policies are encouraged to visit:

http://www.elsevier.com/copyright 


\title{
Motion and shape of bubbles rising through a yield-stress fluid
}

\author{
Darek Sikorski $^{\mathrm{a}}$, Hervé Tabuteau ${ }^{\mathrm{a}, \mathrm{b}}$, John R. de Bruyn ${ }^{\mathrm{a}, *}$ \\ ${ }^{a}$ Department of Physics and Astronomy, University of Western Ontario, London, Ontario, Canada N6A 3K7 \\ ${ }^{\mathrm{b}}$ Laboratoire des Colloïdes, Verres, et Nanomatériaux, Université Montpellier II, Montpellier, France
}

\section{A R T I C L E I N F O}

\section{Article history:}

Received 30 January 2008

Received in revised form

12 November 2008

Accepted 26 November 2008

\section{Keywords:}

Bubbles

Yield-stress fluid

Yield

\begin{abstract}
A B S T R A C T
We study the velocity and shape of air bubbles rising through a transparent yield-stress fluid. The bubbles are small enough compared to the experimental vessel that effects of walls are weak. We find that the terminal rise velocity of the bubbles increases approximately linearly with bubble radius over the range of volumes accessible in our experiments. We observe bubble motion only when the bubbles are larger than a certain critical radius. In terms of a dimensionless yield parameter $Y$, the ratio between the force due to the yield stress and the buoyant force, we observe bubble motion only for $Y \lesssim 0.50 \pm 0.04$. The bubbles are non-spherical, having the shape of an inverted teardrop with a rounded head and a cusp-like tail. The cusps may be an indication that elasticity plays a significant role in this system. By fitting the cross-sectional radius of the bubble as a function of the axial coordinate to an empirical function, we study the dependence of the bubble shape on volume and the yield stress of the material.
\end{abstract}

(C) 2008 Elsevier B.V. All rights reserved.

\section{Introduction}

The entrainment and motion of air or gas bubbles in nonNewtonian fluids is important in many applications, including oil extraction, bioreactors, and food processing. From a fundamental viewpoint, the motion of a single bubble through a complex fluid can be viewed as a benchmark problem that provides information about the forces acting on the bubble and the distribution of stresses in the material. There is an extensive literature dealing with the behavior of bubbles in Newtonian fluids [1,2], but despite the importance of the problem, bubbles in non-Newtonian fluids have not been as thoroughly studied and neither their dynamics nor their shape are completely understood. Previous work has been reviewed by Chhabra [3].

In this paper, we study the behavior of bubbles rising through yield-stress fluids. These materials flow only when the applied stress $\tau$ is greater than a yield stress $\tau_{y}$; for $\tau<\tau_{y}$ they behave as soft solids. As a result, while a gas bubble will always rise due to buoyancy through a fluid with zero yield stress, it can remain stationary in a yield-stress fluid if the buoyant force is insufficient to overcome the opposing force due to the yield stress [4-6]. Similarly, a solid sphere will not fall through a yield-stress fluid unless the net gravitational force is large enough to overcome the yield stress [7]. An object moving through a yield-stress fluid will be surrounded by a region of yielded material that extends a certain distance, but

\footnotetext{
* Corresponding author. Tel.: +1 519661 2111x86441.

E-mail address: debruyn@uwo.ca (J.R. de Bruyn).
}

farther from the object, where the local stress no longer exceeds the yield stress, the material will remain solid-like [7].

In a theoretical treatment of the falling-sphere problem, Beris et al. [7] defined a yield parameter $Y$ proportional to the ratio between the force due to the yield stress and the buoyant force. They found for a Bingham fluid that motion occurred only for values of $Y$ less than a yield criterion $Y_{c}$. While recent experimental results [8] for spheres falling through simple (non-aging [9]) yieldstress fluids agree well with theoretical predictions [7,10,11], there are rather few previous papers concerned with the complementary problem of rising bubbles in yield-stress materials [4-6,12-17] or with the displacement of a yield-stress fluid by gas [18-20]. Theoretical treatment of this problem is complicated by the possibility of coupling among the fluid's rheological properties, the shape of the bubble, and its motion, as well as by the nonlinearity of model constitutive relations for viscoplastic fluids. Estimates of the yield criterion $Y_{c}$ at which the buoyant force on the bubble is balanced by the yield stress have been derived by Dubash and Frigaard [5], and the same group has performed experiments on bubbles rising through a cylinder containing Carbopol, a yield-stress polymer gel [6]. Tsamopoulos et al. have carried out a numerical study of the rise of bubbles through a viscoplastic fluid [16] using a regularized form of the Bingham model [21] and obtained detailed results for the bubble shape and rise velocity as functions of the dimensionless parameters which characterize the problem. They found that $Y_{C}$ depended strongly on the degree to which the bubble could be deformed: when the buoyant force was much larger than the force due to surface tension, the bubble took on a bullet-like shape which allowed it to more easily move through the material. Singh and Denn [17] performed two-dimensional finite-element 
calculations of one or more bubbles rising through a Bingham fluid. They found that backflow in the yielded region around a moving bubble affected the bubble's shape, leading to the development of a cusped tail under some conditions. Bubbles with tapered tails were observed in yield-stress fluids in the experiments of Ref. [6], and cusped tails have also been observed in bubbles rising through viscoelastic fluids with no yield stress [22-26].

Here we study the motion and shape of bubbles in Carbopol dispersions with yield stresses of 24 and $34 \mathrm{~Pa}$, somewhat higher than those of the dispersions used in Ref. [6], and in an experimental container large enough that the influence of the walls on both the shape and the dynamics of the bubbles is expected to be small. We find that the terminal speed of the rising bubbles increases approximately linearly with the effective bubble radius (that is, the radius of a spherical bubble with the same volume) above a certain threshold value, while smaller bubbles do not rise. All moving bubbles were observed to have a rounded head and a cusped tail. We were able to fit the shape of all of our bubbles to a simple empirical function, and we study the dependence of the bubbles' shape on their size and on the yield stress of the fluid. In Section 2 we describe our materials and outline the experimental procedure. Our results are presented in Section 3 and discussed in the context of previous work in Section 4.

\section{Experiments and materials}

Carbopol is a family of commercial polymeric materials which, when dispersed in water and neutralized, forms stable, transparent gels with minimal thixotropy [27-29]. The yield stress of Carbopol gels can be conveniently tuned by varying polymer concentration and pH. We used Carbopol ETD 2050, which forms gels that are particularly clear and so very well-suited for visualization experiments. Two samples with concentrations $c$ of $1.2 \%$ and $1.8 \%$ by weight were prepared as in previous work [8]. The required amount of Carbopol ETD 2050 powder was added to 151 of deionized water in a large tank and allowed to disperse completely. The resulting viscous fluid had a $\mathrm{pH}$ of approximately 3. $1 \mathrm{M} \mathrm{NaOH}$ was then slowly added with continuous mixing to raise the $\mathrm{pH}$ to 6 , by which point the Carbopol dispersion was a pasty, stiff gel with a significant yield stress. Some previous experiments on Carbopol have shown history-dependent results. We have found that extensive mixing of the material allows us to obtain reproducible data [8]; to achieve this, the samples were mixed thoroughly with a propeller-blade mixer for up to $12 \mathrm{~h}$ a day for a week following the addition of the $\mathrm{NaOH}$. The Carbopol was then carefully poured into the experimental vessel. The fluid was thoroughly remixed several times over the course of the measurements to retain the homogeneity of the material and to eliminate any bubbles remaining in the fluid from previous runs. Evaporation was prevented by keeping the gel container covered except when experiments were in progress.

The experiments were performed in a Plexiglas container $80 \mathrm{~cm}$ high, having a square cross-section with sides $12.9 \mathrm{~cm}$ long. Single bubbles were injected through a hole centered in the bottom of the vessel by manually depressing the plunger of a calibrated syringe filled with air and connected to the hole by a length of fine plastic tubing. A $3 \mathrm{~cm}^{3}$ syringe was used to produce bubbles with nominal volumes $V$ (as measured from the syringe) ranging from 0.1 to $2.5 \mathrm{~cm}^{3}$, and a $60-\mathrm{cm}^{3}$ syringe for bubbles with $V>2.5 \mathrm{~cm}^{3}$. The range of bubble sizes that could be studied with our apparatus was limited at both high and low volumes. Bubbles smaller than a fluid-dependent minimum volume were not sufficiently buoyant to detach from the inlet tube, while bubbles larger than a certain maximum volume broke up during the injection process to form two or more smaller bubbles. For $c=1.2 \%$, bubbles with $V$ in the range $0.4-12 \mathrm{~cm}^{3}$ could be studied, while for $c=1.8 \%, 0.6<V<34 \mathrm{~cm}^{3}$.
In all cases, multiple trials were carried out for each nominal bubble volume. A video camera capable of recording images at up to 500 frames per second was focused on a region roughly $30 \mathrm{~cm}$ high starting $10 \mathrm{~cm}$ above the injection hole. Preliminary experiments showed that by the time they entered this field of view, the bubbles were moving at a constant terminal velocity. Recording was triggered automatically by an electronic motion detector when a bubble entered the field of view. The resolution of our images was roughly $1.7 \mathrm{~mm} /$ pixel, limited by the desire to track the motion of the bubbles over a reasonable distance to obtain accurate velocity data. The largest bubbles studied in this work had a maximum cross-sectional radius $R_{\max }$ of $1.6 \mathrm{~cm}$. The perpendicular distance $R_{c}$ from the center of the container to the walls was $6.5 \mathrm{~cm}$, so the ratio $R_{\max } / R_{c} \leq 0.25$ in all cases. We can also consider the ratio $R_{\text {eff }} / R_{c}$, where $R_{\text {eff }}=\left(3 V_{b} / 4 \pi\right)^{1 / 3}$ is the radius of a spherical bubble with the same volume $V_{b}$. For our experiments, $R_{\mathrm{eff}} / R_{c} \leq 0.28$. For these values of the size ratio, wall effects would be expected to contribute at most about $15 \%$ to the drag on a sphere moving through a yield-stress fluid [30]. The effect of walls on bubble motion has not previously been measured but is likely to be of a similar magnitude.

Video images of the moving bubbles were analyzed using commercial image analysis software. Bubble speed was determined by tracking the uppermost point of the bubble from frame to frame. The shape of the bubbles was recorded by manually locating each pixel on the surface of the bubble in a given frame. Bubble volume $V_{b}$ was determined by numerically integrating the recorded shape profile, assuming axial symmetry and averaging over the two sides of the bubble. $V_{b}$ was measured from several different video frames for a given bubble to ensure that it was independent of depth and to evaluate the accuracy of the volume determination. The uncertainties in $V_{b}$ were typically $\pm 0.05 \mathrm{~cm}^{3}$, corresponding to about $\pm 2 \%$ for the largest bubbles or $\pm 10 \%$ for the smallest. The values of $V_{b}$ determined from the image analysis were slightly smaller than the nominal volumes $V$ obtained from the syringe, particularly for larger volumes. This is presumably due to the fact that a small amount of air remains attached to the inlet tube when the bubble pinches off, making $V$ larger than the actual bubble volume. As a result we use $V_{b}$ as the bubble volume in what follows.

The rheological properties of the Carbopol dispersions were measured with an Ares RHS controlled-strain rotational rheometer using a cone-and-plate geometry. Fine emery paper was affixed to each tool surface to minimize wall slip. The flow curve was determined from measurements of the stress $\tau$ under steady shear as a function of shear rate $\dot{\gamma}$, and the elastic and viscous moduli, $G^{\prime}$ and $G^{\prime \prime}$ respectively, were measured as a function of frequency and strain amplitude by applying oscillatory shear. The results of these rheometrical measurements are illustrated in Fig. 1, and show behavior consistent with previous studies on similar yieldstress gels $[8,28]$. The flow curves were well-described by the Herschel-Bulkley model,

$\tau=\tau_{y}+\kappa \dot{\gamma}^{n}$

where the yield stress $\tau_{y}, \kappa$, and the power law index $n$ were treated as fitting parameters. The results of these fits as well as the zero-frequency elastic modulus $G^{\prime}(0)$ for both experimental fluids are given in Table 1. Our experience with Carbopol dispersions is that they do not age significantly over reasonable time scales, but nonetheless the experiments described below were completed

Table 1

Rheological properties of the Carbopol suspensions used in the experiments.

\begin{tabular}{llllr}
\hline$c(\%)$ & $\tau_{y}(\mathrm{~Pa})$ & $\kappa\left(\mathrm{Pa} \mathrm{s}^{n}\right)$ & $n$ & \multicolumn{1}{c}{$G^{\prime}(0)(\mathrm{Pa})$} \\
\hline 1.2 & $24.1 \pm 0.8$ & $13.7 \pm 0.4$ & $0.528 \pm 0.001$ & $80.9 \pm 1.9$ \\
1.8 & $33.5 \pm 0.4$ & $10.5 \pm 0.3$ & $0.545 \pm 0.006$ & $120.5 \pm 3.6$ \\
\hline
\end{tabular}



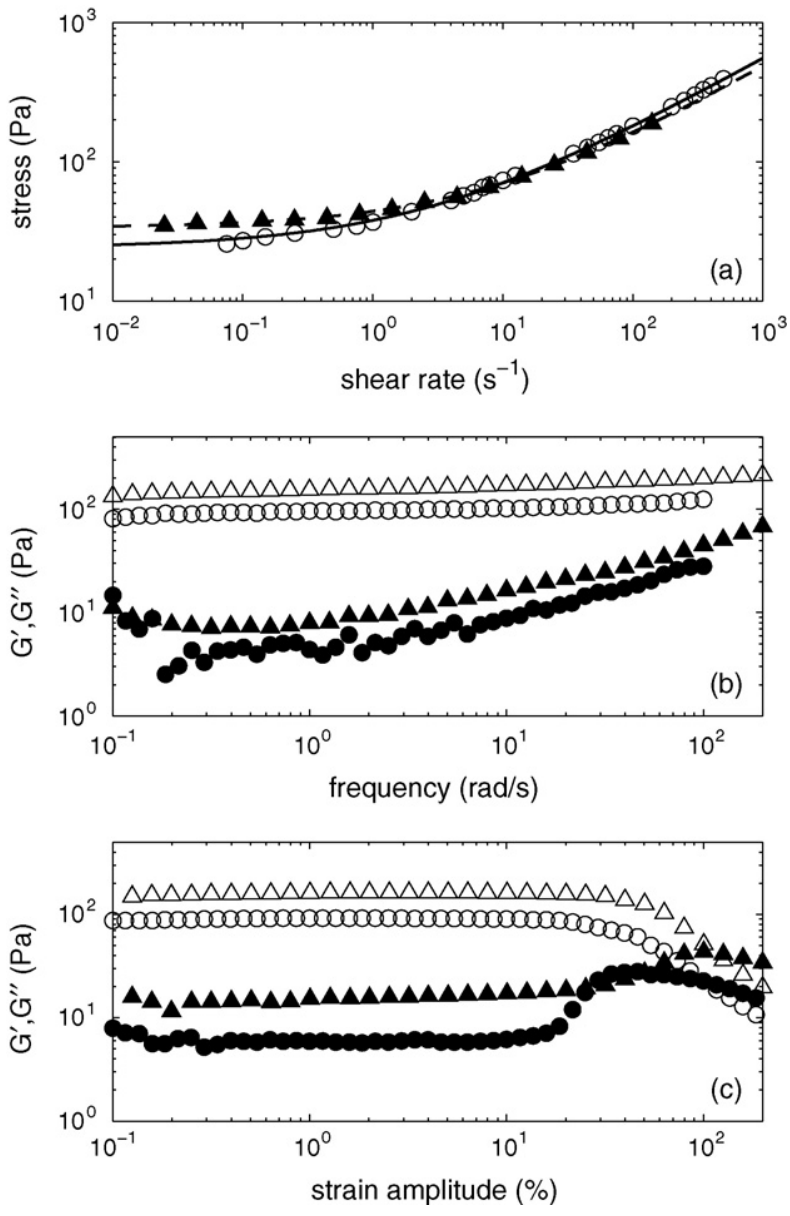

Fig. 1. Rheological properties of the two Carbopol samples used in the experiments. Circles are for $c=1.2 \%$ and triangles for $c=1.8 \%$. (a) Steady-state stress plotted against shear rate. The lines are least-squares fits to the Herschel-Bulkley model, Eq. (1). The elastic and viscous moduli $G^{\prime}$ (open symbols) and $G^{\prime \prime}$ (solid symbols) measured under oscillatory shear are plotted as a function of angular frequency at a strain amplitude of $1 \%$ (which is within the linear regime) in (b) and as a function of strain amplitude at a frequency of $6.28 \mathrm{rad} / \mathrm{s}$ in (c).

within a few days of the rheometrical characterization to ensure that changes in the material properties were not significant.

\section{Results}

Once the injected bubbles separated from the inlet tubing, they accelerated upwards, reaching a constant terminal speed $v_{0}$ by the time they entered the field of view of the video camera. Fig. 2(a) shows the terminal speed of the rising bubbles as a function of their volume $V_{b}$ for both concentrations. The uncertainties in the individual velocity and volume measurements are smaller than the plotted symbols, so the scatter in the data must be a result of uncontrolled variations in the sample from run to run that arise despite our careful remixing procedure. Fig. 2(b) shows the same data plotted as a function of the effective radius $R_{\text {eff }}$. This figure shows clearly that bubbles smaller than a certain critical radius (or volume) do not have sufficient buoyancy to rise against the yield stress of the material. Within the experimental scatter, we can describe the dependence of $v_{0}$ on $R_{\text {eff }}$ by straight lines for each fluid. The fits shown as dashed lines on Fig. 2(b) give critical radii $R_{\text {eff }, c}=0.42 \pm 0.05 \mathrm{~cm}$ for $c=1.2 \%$ and $R_{\mathrm{eff}, c}=0.52 \pm 0.05 \mathrm{~cm}$ for $c=1.8 \%$, corresponding to critical volumes of $0.31 \pm 0.11$ and $0.59 \pm 0.18 \mathrm{~cm}^{3}$, respectively. The same fits are plotted in terms of $V_{b}$ in Fig. 2(a).
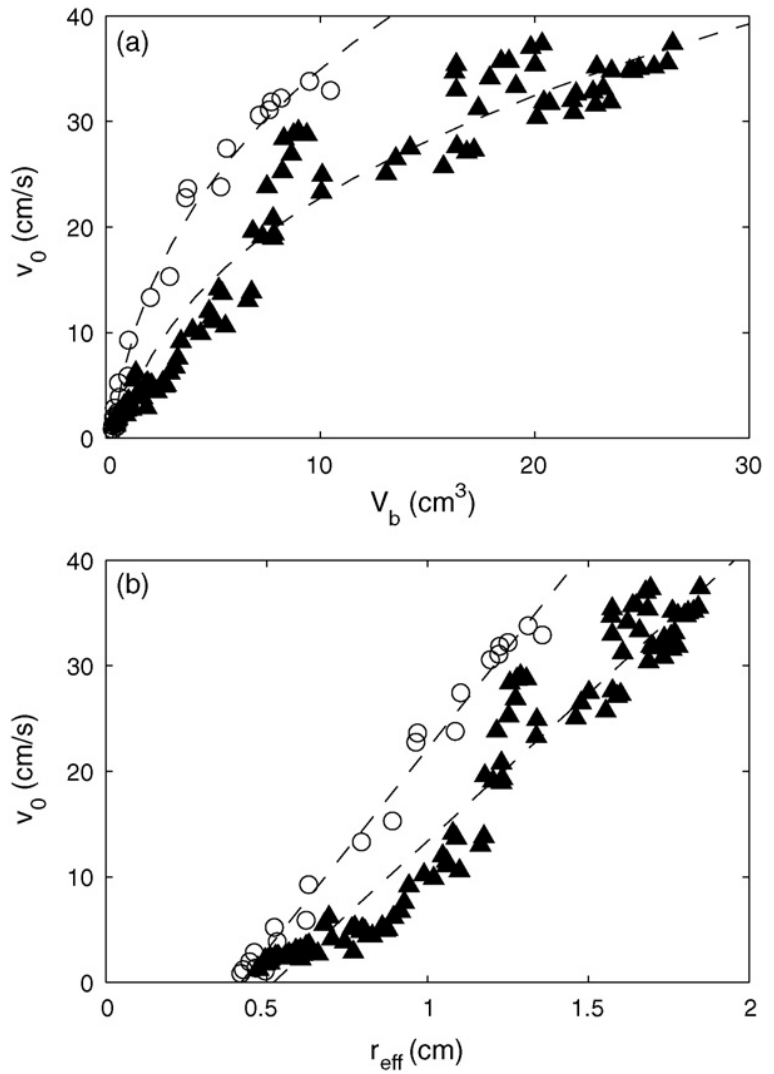

Fig. 2. Terminal bubble rise velocity vs. volume (a) and effective bubble radius $R_{\mathrm{ef}}$ (b) for two Carbopol suspensions. Circles are for $c=1.2 \%$ and triangles for $c=1.8 \%$. The dashed lines in (b) are fits of the data to straight lines; the same fits are plotted in terms of volume in (a).

Fig. 3 shows the same velocity-volume data plotted in dimensionless form. Here we plot the generalized Reynolds number,

$R e=\frac{2 \rho v_{0} R_{\max }}{\mu}$,

against the yield parameter

$Y=\frac{2 \pi \tau_{y} R_{\max }^{2}}{\rho g V_{b}}$.

Here $R_{\max }$ is the maximum radius of the bubble, determined from the video images as explained below. $g$ is the acceleration due to

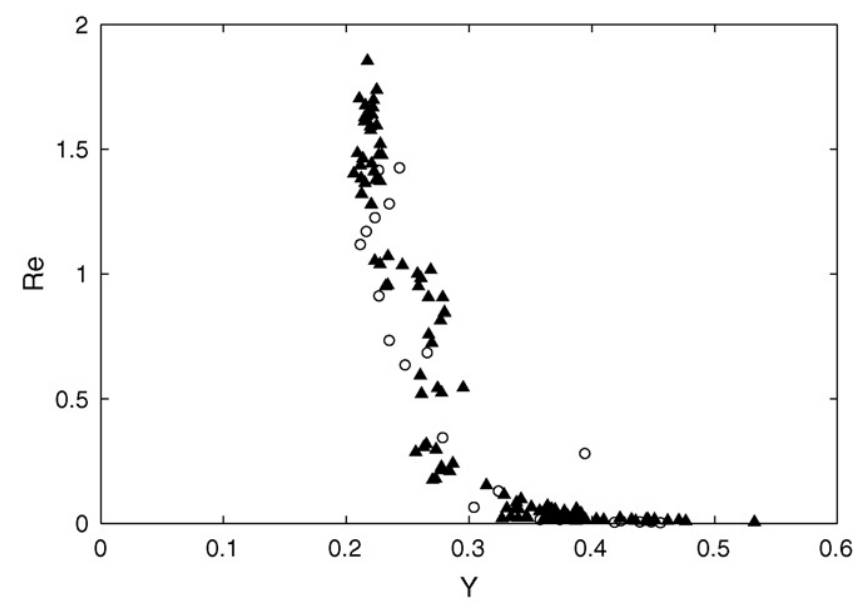

Fig. 3. The generalized Reynolds number Re plotted against the yield parameter $Y$ defined in Eq. (3). Circles are for $c=1.2 \%$ and triangles for $c=1.8 \%$. 
gravity, $\rho$ is the fluid density (which is much larger than that of the air in the bubble) and $\mu=\tau / \dot{\gamma}$ is the viscosity of the material, determined from Eq. (1) with $\dot{\gamma}$ taken to be $v_{0} / R_{\max }$. The data for the two values of $\tau_{y}$ collapse onto a single curve when plotted in this way. No bubble motion is observed for $Y>Y_{c} \approx 0.53$. The dependence of $R e$ on the yield parameter seen in Fig. 3 is qualitatively similar to that predicted numerically for a Bingham fluid (described by Eq. 1 with $n=1$ ) in Fig. 17 of Ref. [16], although we note that other dimensionless quantities (the Bond number and Archimedes number; see Section 4) which are held constant in the numerical work in fact vary in our experiments. Note that our definition of $Y$ is the same as that used by Beris et al. [7], using $R_{\max }$ in the numerator, but differs slightly from that used by Dubash and Frigaard $[5,6]$ and Tsamopoulos et al. [16]. This is discussed further in Section 4 below.

The bubbles were in general far from spherical in shape. For low volumes, they were relatively long and slender with a rounded head and a somewhat conical tail. As the volume increased, the head of the bubble became slightly more pronounced while the profile of the tail showed more concavity. Within the resolution of our images, all bubbles studied appeared to have a tail ending in a cusp.

We determined the cross-sectional radius $R$ of the bubble as a function of the axial coordinate $z$ from the recorded images. To characterize the variation of bubble shape with volume, we plot the maximum radius $R_{\max }$ (averaged over the two sides of the bubble) against $V_{b}$ in Fig. 4 . For a spherical bubble, $R_{\max }$ would be the same

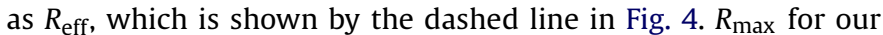
bubbles is always smaller than this, but shows a slightly more rapid increase with volume over the range of our experiments-a fit to the data for $c=1.8 \%$ gives $R_{\max }=(0.428 \pm 0.002) V_{b}^{0.405 \pm 0.002}$. The inset to Fig. 4 shows the aspect ratio $L / R_{\max }$, where $L$ is the total length of the bubble determined from the video images, plotted as a function of $V_{b}$. Our values of $L / R_{\max }$ lie in the range from 4.2 to 10.3 for $c=1.8 \%$, decreasing as $V_{b}$ increases. For a given bubble volume, $L / R_{\max }$ is smaller (i.e., the bubble is closer to spherical) in the fluid with the lower yield stress, consistent with previous results in Carbopol dispersions with a much lower $\tau_{y}[6]$ and also with recent numerical calculations [16].

We found that $R(z)$ could be well described by the functional form

$R(z)=A z^{B}(C-z) \mathrm{e}^{-D z}$

over the full range of volumes studied. Here $A, B, C$, and $D$ are fitting parameters and the head of the bubble is at $z=0$. $C$ corresponds

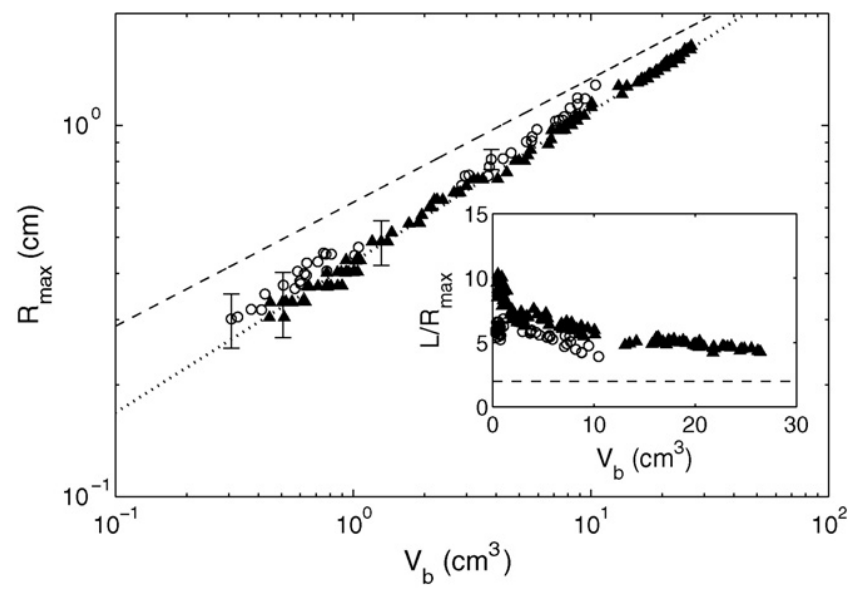

Fig. 4. The relationship between the maximum bubble radius $R_{\max }$ and the bubble volume $V_{b}$. Circles are for $c=1.2 \%$ and triangles are for $c=1.8 \%$. The dashed line shows the radius of a spherical bubble, and the dotted line is a power-law fit to the data for $c=1.8 \%$. The inset shows the aspect ratio $L / R_{\max }$ as a function of $V_{b}$, with the dashed line indicating the value of 2 for a spherical bubble.

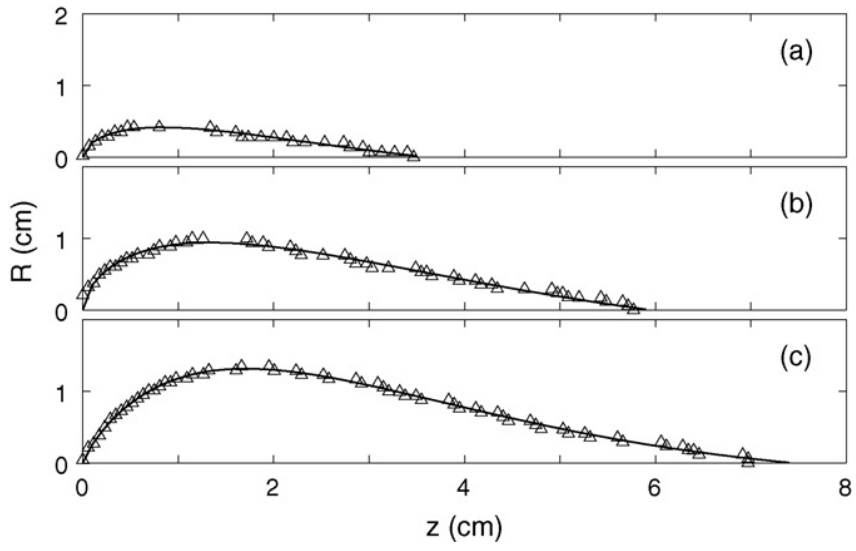

Fig. 5. The measured profiles of bubbles with $c=1.8 \%$ and (a) $V_{b}=0.86 \mathrm{~cm}^{3}$, (b) $7.79 \mathrm{~cm}^{3}$ and (c) $16.26 \mathrm{~cm}^{3}$ are shown by the symbols. The lines are fits of the profiles to Eq. (4).

to the overall length of the bubble. The exponent $B$ characterizes the shape of the head of the bubble, while the exponential decay determines the shape of the cuspy tail. Typical bubble profiles fitted to Eq. (4) are shown in Fig. 5; the quality of the fits is uniformly excellent.

The parameters obtained by fitting Eq. (4) to the measured bubble profiles for $c=1.8 \%$ are plotted against the bubble volume $V_{b}$ in Fig. 6 . The coefficient $A$ shows a weak linear increase as bubble volume increases (shown by the dashed line in Fig. 6(a)). $A$ is approximately a factor of 1.7 larger (but also significantly more scattered) for the lower concentration sample. The exponent $B$ is close to 0.5 for the smallest bubbles, corresponding to a locally parabolic bubble profile, but increases with a weak power-law dependence on $V_{b}$. The fit shown in Fig. 6(b) gives $B=(0.52 \pm 0.01) V_{b}^{0.13 \pm 0.01}$ (with $V_{b}$ in $\mathrm{cm}^{3}$ ). The values of $B$ for $c=1.2 \%$ show the same weak powerlaw increase with $V_{b}$ within our uncertainties, but are on average about 33\% larger. The bubble length $C$ also increased as a power-law in $V_{b}$, with a fit for $c=1.8 \%$ giving $C=(3.76 \pm 0.04) V_{b}^{0.208 \pm 0.004} \mathrm{~cm}$ as shown in Fig. 6(c). The values of $C$ for the lower concentration were the same within the experimental scatter and for both concentrations agreed with bubble lengths $L$ determined directly from the images. Finally, the inverse decay length $D$ appeared to be independent of volume and equal to $0.22 \pm 0.08 \mathrm{~cm}^{-1}$ for $c=1.8 \%$ and $0.5 \pm 0.2 \mathrm{~cm}^{-1}$ for $c=1.2 \%$.

\section{Discussion}

An object will rise or fall through a yield-stress fluid when the net gravitational force is sufficient to overcome the opposing force due to the yield stress. In terms of the dimensionless yield parameter $Y$ defined in Eq. (3) above, the bubble will move if $Y$ is less than some critical value $Y_{c}$, at which point the upward buoyancy force overcomes the force due to the yield stress. As noted above, our definition of $Y$ follows that of Ref. [7], with the maximum bubble radius used in the calculation of the force due to the yield stress in the numerator. Dubash and Frigaard $[5,6]$ and Tsamopoulos et al. [16] used a slightly different definition of $Y$ based on $R_{\text {eff }}$ rather than $R_{\max }$ and differing by an additional numerical factor of 1.5 . The difference is small, and our results are qualitatively the same whichever definition we use. We prefer the definition we have used because it captures more of the physics involved: the drag force will depend on the actual cross-sectional area of the bubble, while the buoyancy force will be proportional to the bubble volume. Since, as we have shown, the yield stress affects the shape of the bubble, using $R_{\text {eff }}$ in the definition of $Y$ might not capture all of the yield-stress dependence in the problem. Defining $Y$ in terms of the 

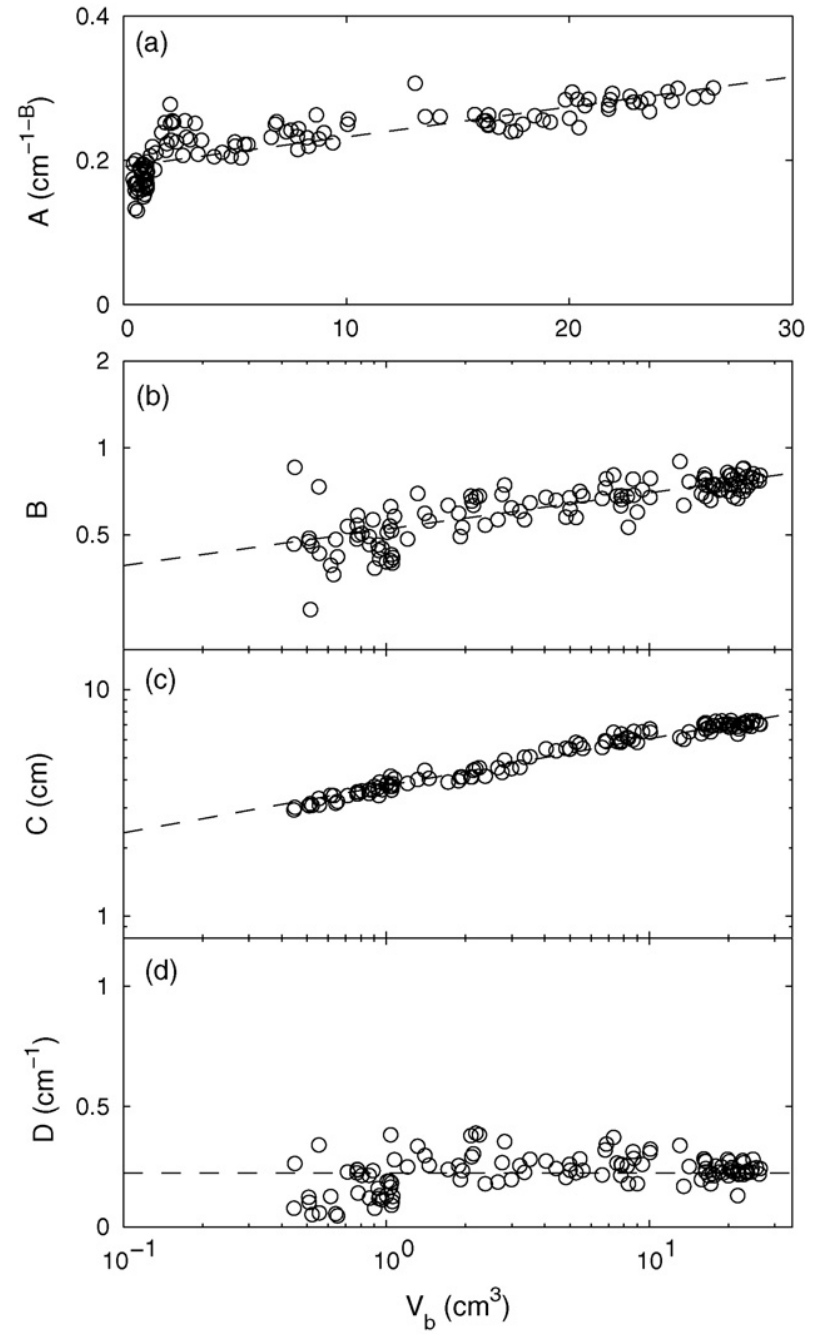

Fig. 6. The parameters $A-D$ obtained from fits of the measured bubble profiles to Eq. (4) for a Carbopol concentration $c=1.8 \%$ are plotted as a function of $V_{b}$ in graphs (a)-(d), respectively. The uncertainties in the parameter values are typically in the order of the scatter in the data. In each case the dashed lines are fits to the data discussed in the text.

actual cross-sectional area of the bubble through the use of $R_{\max }$, as we do, leads to a slightly better collapse of the data and slightly better agreement between the values of $Y_{c}$ obtained for the two fluids. The highest value of $Y$ measured is 0.46 for $c=1.2 \%$ and 0.53 for $c=1.8 \%$. Taking an average gives $Y_{c}=0.50 \pm 0.04$ as an estimate of the critical yield parameter for bubbles rising through Carbopol. (For comparison, defining $Y=\tau_{y} / \rho g R_{\text {eff }}$ as in Refs. [6,16] gives maximum values of $Y$ of 0.59 and 0.72 , respectively.) The small difference between the highest values of $Y$ observed for the two concentrations likely reflects the difficulty in experimentally studying small bubbles which approach the critical condition, and not a real dependence on $\tau_{y}$; we note that any such dependence would be unexpected based on current theory.

Beris et al. [7] calculated $Y_{c}$ for a solid sphere in a Bingham fluid to be 0.143 . This value has recently been confirmed experimentally for spheres falling through Carbopol [8]. $Y_{C}$ could be quite different for bubbles because of their non-spherical shape and because of the different boundary conditions at the surface of the bubble. In fact all of our measured values of $Y$ are higher than the value of $Y_{c}$ for a solid sphere. Dubash and Frigaard $[5,6]$ determined an upper bound for $Y_{C}$ of $L /\left(2^{3 / 2} R_{\text {eff }}\right)$ for axisymmetric bubbles which is a factor of four to ten larger than our experimental values. On the other hand, the same authors [5] also used a comparison principle to obtain an esti- mate of $Y_{C}=\sqrt{3} / 2=0.87$ (using our definition of $Y$ ) for a spherical bubble, only slightly higher than the experimental result (although of course our bubbles were not spherical). Tsamopoulos et al. [16] calculated $Y_{c}$ to be 0.21 (again using our definition of $Y$ ) for bubbles rising through a Bingham fluid. This is somewhat smaller than the value we observe experimentally. The quantitative differences between the experimental and theoretical values of $Y_{C}$ are likely due to the fact that the shapes of the bubbles calculated in Ref. [16], or assumed in Ref. [5], are quite different from the experimental shapes.

Tsamopoulos et al. [16] identify several other dimensionless numbers which characterize this system. These include the Bond number $B o$, the ratio of the gravitational force to that due to surface tension:

Bo $=\rho g R_{\mathrm{eff}}^{2} / \sigma$,

where $\sigma$ is the surface tension, and the Archimedes number $A \mathrm{r}$, the ratio of gravitational forces to viscous forces:

$A r=\rho^{2} g R_{\mathrm{eff}}^{3} / \mu^{2}$.

The Bingham number $B n$, the ratio of yield stress to viscous stresses, is analogous to $Y$ for this system $[6,16]$. In Fig. 7, we show the range of Re, $Y$, and Bo covered by our experiments for the 1.8\% Carbopol dispersion. In calculating Bo, we have assumed $\sigma=0.035 \mathrm{~N} / \mathrm{m}$, although to our knowledge this property has not been measured (Ref. [6] used $\sigma=0.070 \mathrm{~N} / \mathrm{m}$, the value for water, but this is certainly an overestimate). The values of $A r$ are not shown but overlap closely those of $R e$ and show essentially the same volume dependence, ranging from $10^{-2}$ for small bubbles to 3 for large bubbles. Bo ranges from about 3 to 80 , indicating that the effects of surface tension are small compared to buoyancy over the full range of our experiments. This is consistent with the fact that our bubbles are strongly non-spherical. Clearly all of these parameters vary with bubble size, so that in practice it is difficult to perform experiments in which any one of these parameters is varied while others are held constant.

For bubbles that are large enough to overcome the yield stress, the terminal rise velocity increased with bubble volume over the full range of volumes studied and for both concentrations. Fits to our data gave critical volumes $V_{c}$ of $0.31 \pm 0.11 \mathrm{~cm}^{3}$ for the fluid with the lower yield stress and $0.59 \pm 0.18 \mathrm{~cm}^{3}$ for the higher yield-stress material. These are close to the minimum bubble volumes studied in each case: $(0.53 \pm 0.05) \mathrm{cm}^{3}$ and $(0.60 \pm$ $0.09) \mathrm{cm}^{3}$, respectively. The value of $V_{c}$-and hence the buoyant

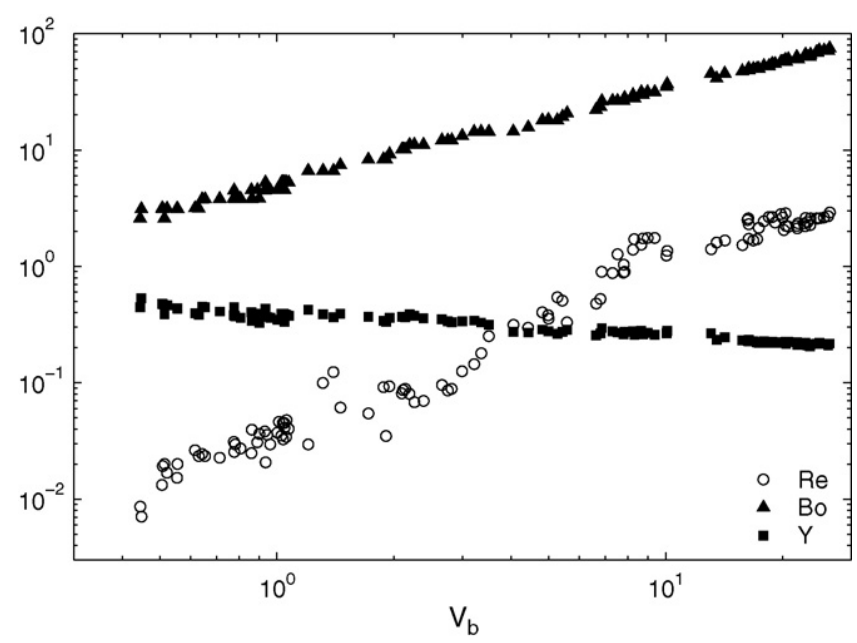

Fig. 7. The range of the dimensionless numbers $R e, B o$, and $Y$ covered in the experiments with $c=1.8 \%$. The numbers are plotted as a function of bubble volume. 
force needed to overcome the yield stress-increases with $\tau_{y}$, as expected.

For $R_{\mathrm{eff}}>R_{c}$ the rise speed of our bubbles was approximately linear in radius. The measurements of Ref. [6] also showed an increase in rise velocity for small bubbles, but followed by a peak and then a regime in which the rise velocity was independent of bubble size for bubbles which nearly filled the container. A similar peak was observed in Ref. [14]. This complex behavior clearly indicates that the walls have a significant effect on the bubble motion for $R / R_{c} \gtrsim 0.5$. This is to be expected from previous studies of the motion of solid spheres through yield-stress fluids [30,31]. No such peak or plateau regime was observed in the present work, in which $R_{\text {eff }} / R_{C}$ is always less than 0.28 and wall effects are much less significant.

Our bubbles do not tend towards spherical at small volumes, as would be the case for a Newtonian fluid, but rather become effectively less spherical: smaller bubbles are relatively longer and more slender than larger bubbles. This indicates that forces due to the yield stress are at least as important as surface tension for small bubbles. The difference between $R_{\max }$ and $R_{\text {eff }}$, which is only $15 \%$ for our largest bubbles, rises to $40 \%$ for the smallest, suggesting that fluid properties rather than wall effects play the dominant role in determining the bubble shape. Our bubbles have aspect ratios $L / R_{\max }$ ranging from a maximum of about 10 for small bubbles to four for large bubbles, with bubbles in the lower yield-stress fluid having lower $L / R_{\max }$. The bubbles of Ref. [6], in fluids with substantially lower yield stress, had aspect ratios ranging from 2 to 5 . This suggests that the yield stress has a systematic influence on the shape of the bubble, and this is made explicit by the experimentally observed dependence on $\tau_{y}$ of the parameters in Eq. (4): the head of the bubble, the curvature of which is characterized by the parameter $B$, is flatter, while the decay length $1 / D$ of the tapered tail is longer for the fluid with the higher yield stress. The increase in aspect ratio with increasing yield stress is consistent with the trend seen in the numerical work of Ref. [16].

Our bubbles have a rounded head and a tapered tail over the range of volumes studied. They are similar in appearance to the smallest bubbles illustrated in Ref. [6], but within our resolution the tails of our bubbles all appear to have a sharp cusp, while some of those shown in Ref. [6] have rounded tails. This difference may be due to the significantly larger yield stress of the fluids used in the present work. Cusped bubbles have been observed in several types of non-Newtonian fluids, including purely elastic fluids and shearthinning viscoelastic fluids $[3,12,22,32,33]$. It has been suggested on the basis of numerical studies that the cusp in viscoelastic fluids arises due to a strong uniaxial extensional flow or a negative wake behind the rising bubble, resulting from the stretching of polymer molecules [23-26]. The recent numerical work by Tsamopoulos et al. [16], which did not include elasticity, predicts an elongation of the bubbles in the $z$-direction as $\tau_{y}$ increases but not the strongly tapered tails observed here and in Ref. [6]. It was suggested in Ref. [16] that the cusps may be due to the effects of elasticity in the yield-stress case as well as in the polymer case. On the other hand, Singh and Denn observed cusped bubbles in a two-dimensional calculation of bubbles rising through a Bingham fluid. Their model also did not include elasticity, and they attributed the formation of cusps in their simulations to backflow in the yielded region around the bubble.

While the situation is thus not entirely clear, our observation of cusped bubbles in these experiments suggests that elasticity may significantly affect the fluid dynamics of Carbopol. This is in contrast to the typical picture of Carbopol as a near-ideal viscoplastic fluid. We can get an idea of the importance of elastic effects in our experiments by estimating the Deborah number, defined as $D e=\lambda_{r} / \lambda_{f}$, where $\lambda_{r}$ is the elastic relaxation time of the material and $\lambda_{f}$ a characteristic time scale for the flow. We take $\lambda_{f} \approx R_{\mathrm{eff}} / \nu_{0}$ and

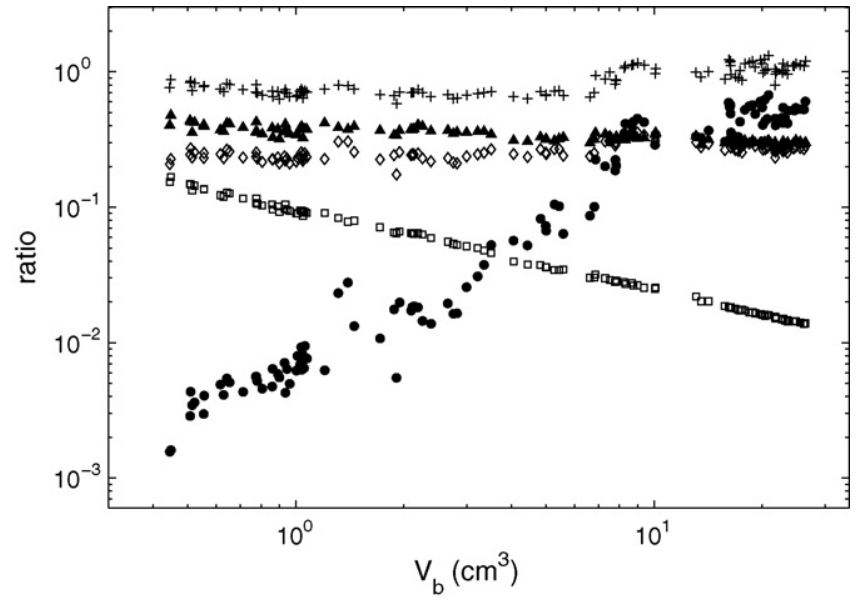

Fig. 8. The ratios of the energy dissipated per unit time by yield stress (solid triangles), inertial forces (solid circles), viscous dissipation (open diamonds), and surface tension (open squares), to that released by buoyancy. The ratio $W_{\text {tot }} / W_{b}$ is shown by the crosses. Data for $c=1.8 \%$ are shown.

$\lambda_{r} \approx \mu / G^{\prime}$, where $G^{\prime}$ is determined at the characteristic frequency $1 / \lambda_{f}$. We find $0.3 \lesssim D e \lesssim 0.5$ for $c=1.8 \%$, and $0.5 \lesssim D e \lesssim 0.9$ or $c=1.2 \%$. Thus while elastic effects will not be dominant in these experiments, they will be present. In fact, cusps have been observed in numerical simulations for De of order one [23,24,26], which is consistent with the possibility that elasticity could be the cause of the cusped tails we observe. Further experimental and numerical studies are required to clarify the issue.

In Ref. [6], Dubash and Frigaard estimated the contributions to the energy budget of the bubbles due to buoyancy, yield stress, inertia, viscosity, and surface tension, taking into account wall effects and assuming a cylindrical bubble with hemispherical end caps. We have repeated their analysis for our bubbles using Eqs. (13)-(17) from Ref. [6], both for our actual values of $R / R_{C}$ and in the limit that $R / R_{c}$ goes to zero. The total dissipation calculated for the two cases differed by at most $6 \%$, consistent with the expected low level of wall effects in our experiment. In Fig. 8, we have plotted the ratios of the energy dissipated per unit time by the "downward" forces due to yield stress $W_{y}$, inertia $W_{i}$, viscosity $W_{v}$, and surface tension $W_{s}$ to the energy released per unit time by the "upward" buoyancy force $W_{b}$, as a function of bubble volume for $c=1.8 \%$. The results for the lower concentration are similar. The ratio $W_{\text {tot }} / W_{b}$, where $W_{\text {tot }}=W_{y}+W_{i}+W_{v}+W_{s}$, is also shown. At small volumes the bubble speed is low so the inertial contribution is negligible, while yield stress, viscosity, and surface tension all contribute at a similar level to the energy budget. This is consistent with the observed non-spherical shape of the bubbles. As $V_{b}$ and the bubble speed increase, inertial effects become correspondingly larger, dominating for the largest bubbles studied. The relative contributions due to viscosity and yield stress remain almost constant as $V_{b}$ increases, while that due to surface tension decreases. For a bubble moving at a constant speed, one would expect the energy released by buoyancy to be completely dissipated by the effects included in $W_{\text {tot }}$, and, despite the fact that the expressions used in the calculations [6] are approximate, we find $W_{\text {tot }} / W_{b}$ to be approximately constant within our scatter and close to one over the range of volumes studied; on average $W_{\text {tot }} / W_{b}=0.86 \pm 0.19$ for $c=1.8 \%$ and $1.2 \pm 0.3$ for $c=1.2 \%$.

\section{Conclusion}

We have examined the behavior of bubbles rising through Carbopol dispersions with yield stress of 24 and $34 \mathrm{~Pa}$ in a system in which wall effects are small. Over the range of bubble volumes 
studied, the rise velocity increases roughly linearly with bubble radius above a critical radius. We find the maximum value of the yield parameter above which the buoyant force is not sufficient to overcome the yield stress to be about $0.50 \pm 0.04$, in qualitative agreement with theoretical predictions. The bubbles have a rounded head and a tapered tail, the latter possibly due to the elasticity of the fluid. Small bubbles are relatively longer and more slender than larger bubbles, reflecting the importance of forces due to the yield stress relative to surface tension forces for small bubbles. Bubbles in the fluid with the larger yield stress have a larger length-to-radius aspect ratio, a flatter head, and a longer tail.

\section{Acknowledgment}

This research was supported by the Natural Science and Engineering Council of Canada.

\section{References}

[1] R. Clift, J.R. Grace, M.E. Weber, Bubbles, Drops and Particles, Academic Press, New York, 1978.

[2] J. Magnaudet, I. Eames, The motion of high-Reynolds number bubbles in inhomogeneous flows, Annu. Rev. Fluid Mech. 32 (2000) 659-708.

[3] R.P. Chhabra, Bubbles, drops, and particles in non-Newtonian fluids, CRC Press, Boca Raton, 2006

[4] S.M. Bhavaraju, R.A. Mashelkar, H.W. Blanch, Bubble motion and mass transfer in non-Newtonian fluids, AIChE J. 24 (1978) 1063-1070.

[5] N. Dubash, I.A. Frigaard, Conditions for static bubbles in viscoplastic fluids, Phys. Fluids 16 (2004) 4319-4330.

[6] N. Dubash, I.A. Frigaard, Propagation and stopping of air bubbles in Carbopol solutions, J. Non-Newtonian Fluid Mech. 142 (2007) 123-134.

[7] A.N. Beris, J.A. Tsamopoulos, R.C. Armstrong, R.A. Brown, Creeping flow of a sphere through a Bingham plastic, J. Fluid Mech. 158 (1985) 219-244.

[8] H. Tabuteau, P. Coussot, J.R. de Bruyn, Drag force on a sphere in steady motion through a yield-stress fluid, J. Rheol. 51 (2007) 125-137.

[9] H. Tabuteau, F.K. Oppong, J.R. de Bruyn, P. Coussot, Drag on a sphere moving through an aging system, Europhys. Lett. 78 (2007) 68007-1-680075-680075.

[10] J. Blackery, E. Mitsoulis, Creeping motion of a sphere in tubes filled with Bingham plastic material, J. Non-Newtonian Fluid Mech. 70 (1997) 59-77.

[11] M. Beaulne, E. Mitsoulis, Creeping motion of a sphere in tubes filled with Herschel-Bulkley fluids, J. Non-Newtonian Fluid Mech. 72 (1997) 55-71.

[12] G. Astarita, G. Apuzzo, Motion of gas bubbles non-Newtonian liquids, AIChE J. 11 (1965) 815.
[13] S. Stein, H. Buggish, Rise of pulsating bubbles in fluids with a yield stress, Z. Angew. Math. Mech. 80 (2000) 827-834.

[14] S.V. Vasil'chenko, A.G. Potapov, Gas bubble dynamics in a viscoelastic-plastic medium, Heat Transfer Res. 27 (1996) 4-8.

[15] Y. Dimakopoulos, J.A. Tsamopoulos, Transient displacement of a viscoplastic material by air in straight and suddenly constricted tubes, J. Non-Newtonian Fluid Mech. 111 (2003) 43-75.

[16] J.A. Tsamopoulos, Y. Dimakopoulos, N. Chatzidai, G. Karapetsas, M. Pavlidis, Steady bubble rise and deformation in Newtonian and viscoplastic fluids and conditions for bubble entrapment, J. Fluid Mech. 601 (2008) 123.

[17] J.P. Singh, M.M. Denn, Interacting two-dimensional bubbles and droplets in a yield-stress fluid, Phys. Fluids 20 (2008) 040901.

[18] A. Lindner, P. Coussot, D. Bonn, Viscous fingering in a yield stress fluid, Phys. Rev. Lett. 85 (2000) 314-317.

[19] A.N. Alexandrou, V. Entov, On the steady-state advancement of fingers and bubbles in a Hele-Shaw cell filled by a non-Newtonian fluid, Eur. J. Appl. Math. 8 (1997) 73-87.

[20] A.N. Alexandrou, V. Entov, S.S. Kolganov, N.V. Kolganova, On bubble rising in a Hele-Shaw cell filled with a non-Newtonian fluid, Eur. J. Appl. Math. 15 (2004) 315-327.

[21] T.C. Papanastasiou, Flows of materials with yield, J. Rheol. 31, 385-404.

[22] L.G. Leal, J. Skoog, A. Acrivos, On the motion of gas bubbles in a viscoelastic liquid, Can. J. Chem. Eng. 49 (1971) 569.

[23] D.S. Noh, I.S. Kang, L.G. Leal, Numerical solutions for the deformation of a bubble rising in dilute polymeric fluids, Phys. Fluids A5 (1993) 1315.

[24] S. Ramaswamy, L.G. Leal, The deformation of a Newtonian drop in the unixial extensional flow of a viscoelastic liquid, J. Non-Newtonian Fluid Mech. 88 (1999) 149.

[25] C. Málaga, J.M. Rallison, A rising bubble in a polymer solution, J. Non-Newtonian Fluid Mech. 141 (2007) 59.

[26] S.B. Pillapakkam, P. Singh, D. Blackmore, N. Aubry, Transient and steady state of a rising bubble in a viscoelastic fluid, J. Fluid Mech. 589 (2007) 215.

[27] Noveon technical data sheet No. 216, www.pharma.noveon.com/literature/ tds/tds216.pdf.

[28] S.J. Curran, R.E. Hayes, A. Afacan, M.C. Williams, P.A. Tanguy, Properties of Carbopol solutions as models for yield-stress fluids, J. Food Sci. 67 (2002) 176-180.

[29] J.M. Piau, Carbopol Gels: Carbopol gels: Elastoviscoplastic and slippery glasses made of individual swollen sponges, J. Non-Newtonian Fluid Mech. 144 (2007) $1-29$.

[30] D.D. Atapattu, R.P. Chhabra, P.H.T. Uhlherr, Wall effect for spheres falling at small Reynolds number in a viscoplastic medium, J. Non-Newtonian Fluid Mech. 38 (1990) 31-42.

[31] J.R. de Bruyn, Transient and steady-state drag in foam, Rheol. Acta 44 (2004) 150-159.

[32] R. Chhabra, D. De Kee (Eds.), Transport Processes in Bubbles, Drops, and Particles, Hemisphere, New York, 1992.

[33] A. Belmonte, Self-oscillations of a cusped bubble rising through a micellar solution, Rheol. Acta 39 (2000) 554-559. 